\title{
RARE Case Report of Primary Gastric Melanoma
}

\author{
K. T. Ashwini, Deepa Mahesh, and M. S. Sandeep
}

\section{ABSTRACT}

Gastric melanomas are usually metastatic, and primary is almost rare entity.

Present case is a rare malignant melanoma of the gastric region with barely reported cases in the literature. A 65 year old male presented with pain abdomen and melena $\mathrm{He}$ was evaluated with relevant investigation. UGI endoscopy and PETCT shows large polypoidal irregular gastric mass in the proximal curvature extending upto gastroesophageal junction. Endoscopic biopsy a ulcero-infiltrating tumour, Individual cells are large, round, having moderately pleomorphic, hyperchromatic nuclei with prominent eosinophilic nucleoli and moderate eosinophilic cytoplasm. Immunohistochemistry of S100 and HMB45 was positive and negative for CK7 and CK20.

Early diagnosis of primary gastric melanoma allows for better prognosis of the patient from the right intervention.

Keywords: Melanoma, primary, Gastric.

\section{INTRODUCTION}

Digestive tract melanomas are rare, typically occurs in anogenital region. Less common location involves oesophagus, stomach, intestine and gallbladder. Primary melanomas are considered as cutaneous neoplasm and extracutaneous presentation is very uncommon and often primary site is difficult to identify.

The pathogenesis of primary mucosal melanoma are environmental, immune, hereditary risk factors and association with other co morbidities, however, with no proven concurrences [1].

\section{CASE REPORT}

A 65 year old male presented with pain abdomen and melena since 15 days. He was evaluated with relevant investigation. UGI endoscopy revealed large, nodular, proliferative, ulcerated, friable fleshy growth seen in the fundus (Fig. 1).
Submitted : March 10, 2021

Published : March 31, 2021

ISSN: $2593-8339$

DOI: $10.24018 /$ ejmed.2021.3.2.763

Dr K. T. Ashwini *

MD, Fellowship in oncopathology

Department of pathology, Apollo Hospital, Bengaluru, Karnataka, India.

(e-mail: drashwinik.t@gmail.com)

Dr. Deepa Mahesh

MD, Department of pathology, Apollo Hospital, Bengaluru, Karnataka, India.

(e-mail: drdeepa_m@apollohospitals.com) Dr. M. S. Sandeep

MD, DM (Gastro)

Senior consultant Gastroenterologist, Apollo Hospital, Sheshadripuram, Bengaluru, India.

(e-mail : sandeepmsdoc@ rediffmail.com)

*Corresponding Author

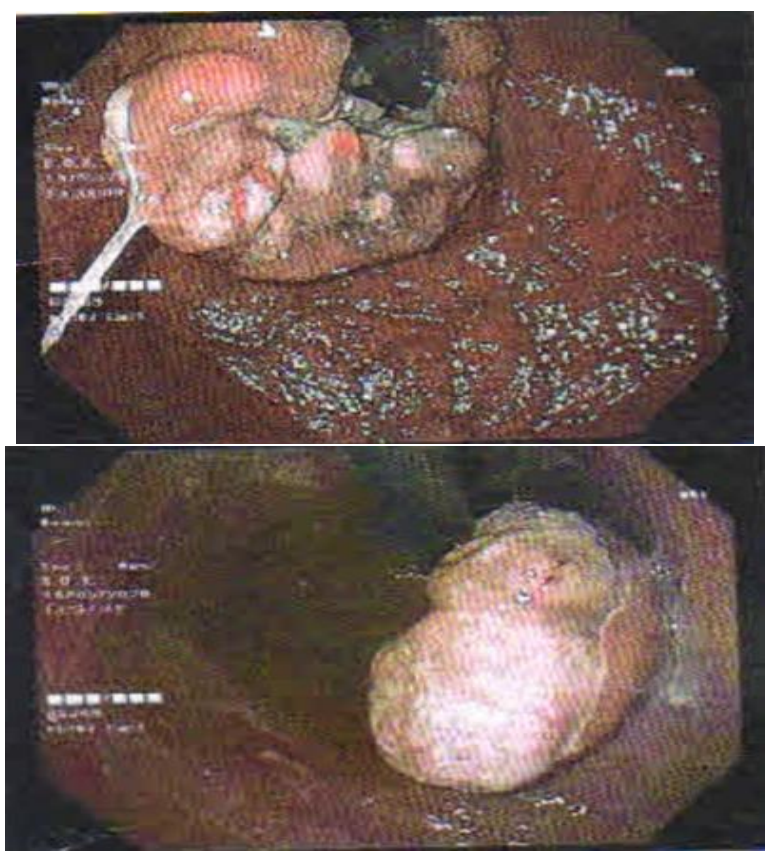

Fig 1. Endoscopic view of nodular proliferative lesion in the fundus.

PETCT shows large polypoidal irregular gastric mass in the proximal curvature extending up to gastroesophageal junction. Endoscopic biopsy was taken and measuring $0.4 \times 0.2 \times 0.1 \mathrm{~cm}$. 


\section{OBSERVATION}

\section{A. Microscopic Findings}

Histological examination showed gastric mucosa with ulcero-infiltrating tumour (Fig. 2), Individual cells are large, round, having moderately pleomorphic, hyperchromatic nuclei with prominent eosinophilic nucleoli and moderate eosinophilic cytoplasm (Fig. 3). Many atypical mitosis noted. Differential diagnosis of poorly differentiated adenocarcinoma and malignant melanoma was offered.

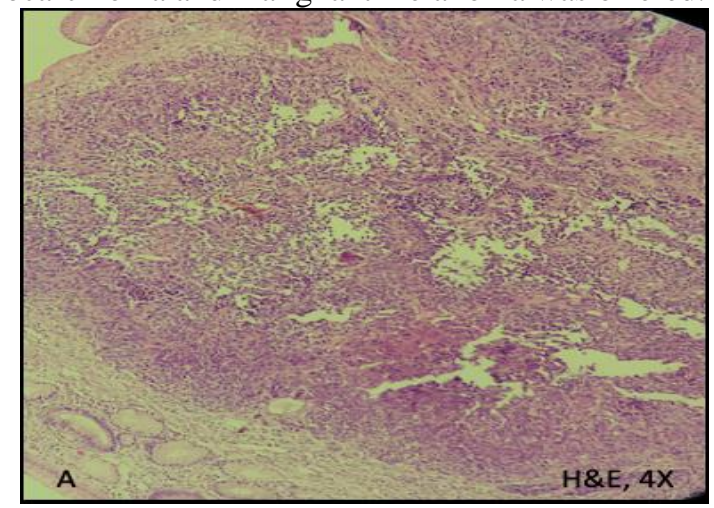

Fig. 2. Histopathological examination of the tumour: Low power view of the ulcerated mucosa with tumour.

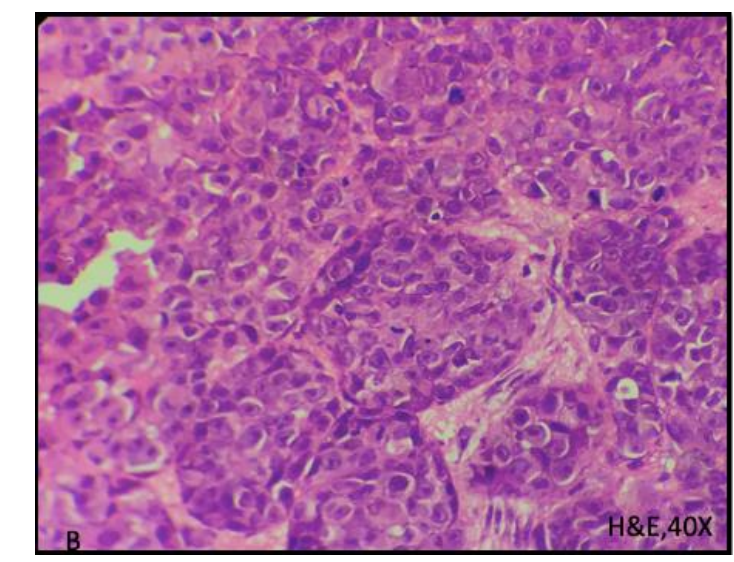

Fig. 3. The cells are round to oval cells with prominent eosinophilic nucleoli and moderate eosinophilic cytoplasm in B. (H and E, 40X).

\section{B. Immunohistochemistry Findings}

The neoplastic cells are positive for S100 (Fig. 4), HMB45 (Fig. 5) and negative for CK7 and CK20 (Fig. 6) and this confirmed the diagnosis of malignant melanoma at rare site.

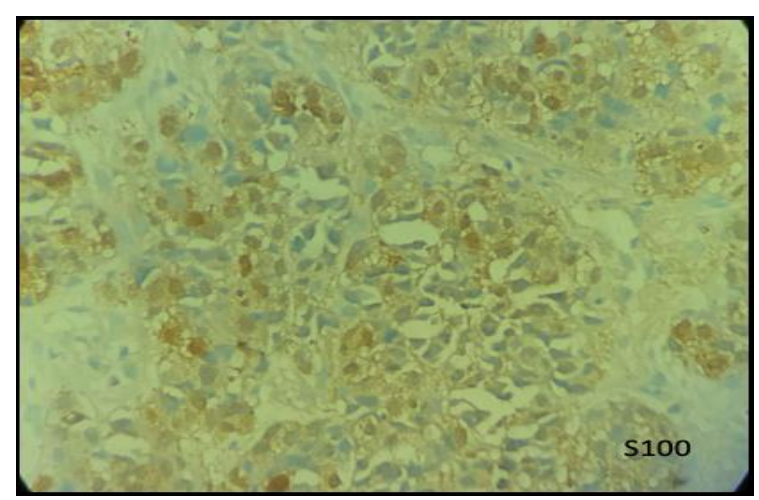

Fig. 4. Immunohistochemical staining: Tumour cells show positive for S100.
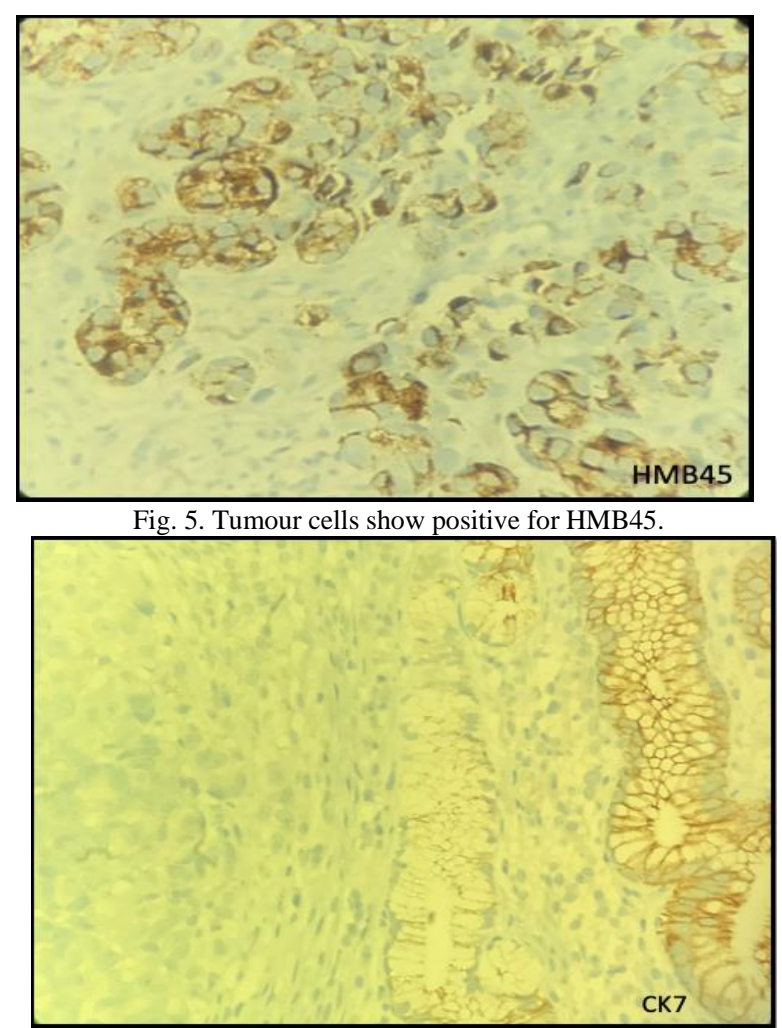

Fig. 6. Tumour cells show negative for CK7.

A final diagnosis of primary malignant melanoma at an unusual site was confirmed.

\section{DISCUSSION}

Melanomas at intestine and stomach are mostly metastasis rather than a primary tumor.

The criteria for diagnosis of primary gastric melanoma include: A lesion in the stomach must be proven by histopathological examination, absence of lesions elsewhere on the body, negative personal history for melanoma and disease-free survival of at least, 12 months after curative surgery [2]-[4]. However in our case patient is on neo adjuvant chemotherapy, follow up is not complete.

Etiology of these primary gastric melanomas is unknown. There are two theories suggests the formation of primary melanoma lesions in the alimentary tract. The theory of possibility is the transformation of nerve cells from the APUD family (amine precursor uptake and decarboxylation) into melanocytes, which then undergo neoplastic transformation [5].

The second theory could be possibility of melanocyte precursor migration into the digestive tract. This hypothesis is based on observations of benign melanosis, which may be accompanied by the development of gastrointestinal tumours [6].

Most common symptoms of primary melanoma are weight loss, upper gastrointestinal bleeding, and anaemia. Upper GI endoscopy and biopsy with positive immunohistochemical stains for S100 protein, Melan-A, and HMB-45 antibodies are crucial role in diagnosis [7], [8]. The tumour in the present case presented with similar clinical scenario and immunohistochemistry positive for S100 and HMB45.

The prognosis of the tumour depends on the factors such as stage of the tumour, failure to perform surgical resection, 
lymph node status, age and other co morbidities. The median survival time is five months [1], [7]-[9].

\section{CONCLUSION}

Early diagnosis of primary gastric melanoma allows for better prognosis of the patient from the right intervention.

\section{ACKNOWLEDGMENT}

The authors express their gratitude to patient and authorities, Apollo hospital Seshadripuram for cooperating in the work.

\section{REFERENCE}

[1] Lagoudianakis EE, Genetzakis M, Tsekouras DK, et al. Primary gastric melanoma: a case report. World J Gastroenterol 2006 July 21; 12(27): 4425-7.

[2] Callaghan GM, Kellher FC, Crowther S, Alakkari A, Ryan BM. A case of primary gastric melanoma exhibiting a rare BRAF V600R mutation. Eur J Case Rep Intern Med. 2018; 5(3): 000749.

[3] Elsayed AM, Albahra M, Nzeako UC, Sobin LH. Malignant melanomas in the small intestine: a study of 103 patients. Am J Gastroenterol. 1996;91:1001-1006.

[4] Chang AE, Karnell LH, Menck HR. The National Cancer Data Base report on cutaneous and noncutaneous melanoma: a summary of 84,836 cases from the past decade. The American College of Surgeons Commission on Cancer and the American Cancer Society. Cancer. 1998;83:1664-1678.

[5] Tabaie HA, Citta RJ, Gallo L, Biondi RJ, Meoli FG, Silverman D (1984) Primary malignant melanoma of the small intestine: report of a case and discussion of the APUD cell concept. J Am Osteopath Assoc 83:374-377.

[6] Horowitz M, Nobrega MM (1998) Primary anal melanoma associated with melanosis of the upper gastrointestinal tract. Endoscopy 30:662665.

[7] Anupama R. Primary gastric melanoma: a rare cause of upper gastrointestinal bleeding. GastroenterolHepatol. 2008; 4(11): 795-7.

[8] Song W, Liu F, Wang S, Shi H, He W, He Y. Primary gastric malignan melanoma: challenge in preoperative diagnosis. Int $\mathrm{J}$ ClinExpPathol. 2014; 7(10): 6826-31.

[9] Augustyn A, Leon ED, Yopp AC. Primary gastric melanoma: case report of a rare malignancy. Rare Tumors. 2015; 7: 46-9. 